\title{
Rationale for hypoxia assessment and amelioration for precision therapy and immunotherapy studies
}

\author{
Mark W. Dewhirst, ${ }^{1}$ Yvonne M. Mowery, ${ }^{1}$ James B. Mitchell, ${ }^{2}$ Murali K. Cherukuri, ${ }^{2}$ and Timothy W. Secomb ${ }^{3}$ \\ 'Radiation Oncology Department, Duke University Medical School, Durham, North Carolina, USA. ${ }^{2}$ Radiation Biology Branch, National Cancer Institute, Bethesda, Maryland, USA. ${ }^{3}$ Department of Physiology, \\ University of Arizona College of Medicine, Tucson, Arizona, USA.
}

$\mathbf{P}$ major focus of cancer therapeutics. This strategy is based on the hope that therapy tailored to the specific molecular features of a patient's cancer will yield superior results, compared with the traditional "one-size-fitsall” therapeutic approach (1). Similarly, modern immunotherapeutic approaches aim to take advantage of establishing a tumor-specific immune response that will eradicate tumor cells within an individual patient and provide lasting antitumor immune memory (2).

Despite promising advances in precision oncology, hypoxia (defined here as partial pressure of oxygen $\left[\mathrm{pO}_{2}\right]<10$ $\mathrm{mmHg}$ ) remains a significant barrier to therapeutic efficacy. Small molecule-targeted agents are susceptible to cellular drug resistance mechanisms, including mechanisms driven by hypoxia $(3,4)$. Spatial and temporal variation in tumor hypoxia (5) may contribute to the heterogeneity in treatment response to precision oncology drug combinations (1).

Hypoxia influences efficacy of immunotherapy via several mechanisms, including both innate and adaptive immunity. For example, hypoxia promotes polarization of macrophages toward the immunosuppressive M2 phenotype (6) and affects NK cell function by reducing expression of the activating receptor NKG2D (7). With respect to the adaptive immune system, hypoxia disturbs the balance between effector $\mathrm{T}$ cells and immunosuppressive regulatory $\mathrm{T}$ cells (8). Hypoxia also interferes with immune checkpoint balance by decreasing MHC class I expression by tumor cells (9), increasing programmed death ligand 1 (PD-L1) expression via the HIF-1 pathway, and promoting secretion of immunosuppressive cytotoxic $\mathrm{T}$ lympho- cyte antigen-1 (CTLA-4) from tumor stromal cells $(10,11)$. Recently it was reported that selective killing of hypoxic cells by TH-302 (evofosfamide) combined with checkpoint blockade greatly enhances immunoreactivity and antitumor effects in several immunotherapy-resistant prostate tumor cell lines (12).

Despite evidence that hypoxia is a confounding factor for both precision therapy and immunotherapy, we were unable to find any precision therapy trials in the NIH ClinicalTrials.gov website that considered hypoxia as a secondary endpoint. Further, only one immunotherapy trial is examining whether hypoxia reduces immunotherapy efficacy to programmed cell death 1 (PD-1) and CTLA-4 blockade with nivolumab and ipilimumab, respectively (NCT03003637). In this trial, ${ }^{18} \mathrm{~F}-\mathrm{HX} 4 \mathrm{PET}$ is used to guide tumor biopsies of hypoxic and normoxic regions for assessment of $\mathrm{T}$ cell infiltrate and effector function before and after immunotherapy. A second trial, NCT03098160, is examining treatment responses when the hypoxic cytotoxin evofosfamide is combined with the CTLA-4 checkpoint inhibitor ipilimumab; however, hypoxia is not assessed in this trial. NCT03575598 will examine effects of immunotherapy with a tyrosine kinase inhibitor, sitravatinib, and PD-1 blockade with nivolumab in patients with head and neck cancer. Changes in hypoxia prior to and after immunotherapy will be assessed using ${ }^{18}$ FAZA PET. With the recent validation of conventional MRI to assess tumor oxygenation status, a trial is underway to monitor hypoxia in head and neck cancer (NCT03646747), but this trial does not involve immunotherapy. The lack of broader attention to hypoxia may be due to the perception that hypoxia is challeng-

Conflict of interest: MWD received an honorarium from Ebix Inc./Oakstone Publishing LLC and is a member of the Scientific Advisory Boards of Innovate Biopharmaceuticals and Siva Therapeutics.

Reference information: J Clin Invest. 2019;129(2):489-491. https://doi.org/10.1172/JCI126044.

ing and/or expensive to measure, while also being difficult to reduce effectively with currently available therapies.

It has been recognized since the 1960s that hypoxia is a potential cause for treatment failure after radiotherapy. Accordingly, many clinical trials have used a range of methods to reduce hypoxia as a means to improve radiotherapeutic or chemoradiotherapeutic outcome. In a meta-analysis, Overgaard and Horsman concluded that data from head and neck cancer trials provided the only clear evidence that reducing hypoxia affected radiotherapy outcome (13). Importantly, hypoxia was not an entry criterion for any of these trials; the trial designs did not account for the possibility that normoxic tumors would not benefit from the hypoxia modification method. The failure of prior hypoxia modification trials with radiotherapy suggests that precision therapeutics and immunotherapy would not benefit from such strategies either.

However, the requirements for hypoxia modification for precision therapeutics and immunotherapy are very different from those for radiotherapy. Hypoxia modification to improve radiotherapy efficacy is only required during the time that treatment is given, because oxygen is required to create stable adducts in damaged DNA that are not easily repaired. In contrast, hypoxia reduction would have to occur chronically over weeks to months of treatment for both precision therapeutics (with daily oral therapy) and immunotherapy with antibodies that block checkpoints from actively inhibiting immune reactivity. These differences in requirement for hypoxia modification open up alternative methods to reduce hypoxia that were not broadly tested with radiotherapy.

We hypothesize that the most efficacious methods to modulate hypoxia in precision therapeutics and immunotherapy trials would be to promote improved microvascular network function, com- 
A

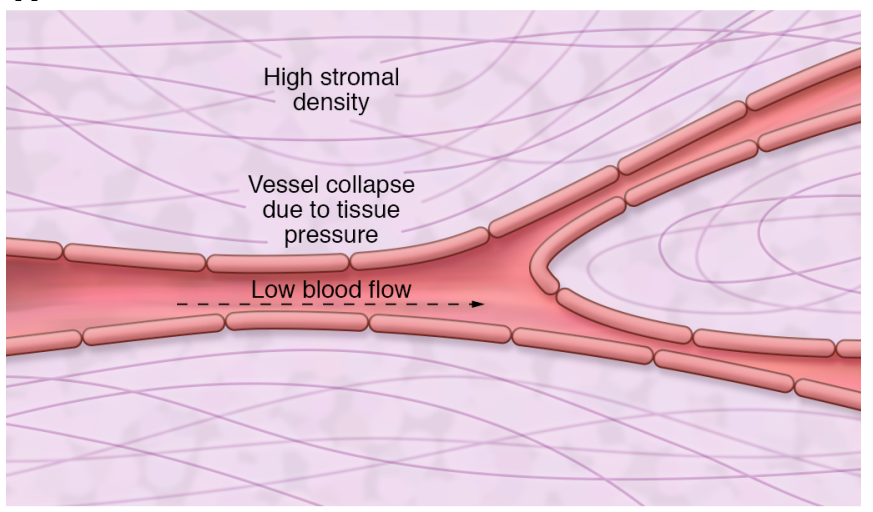

B
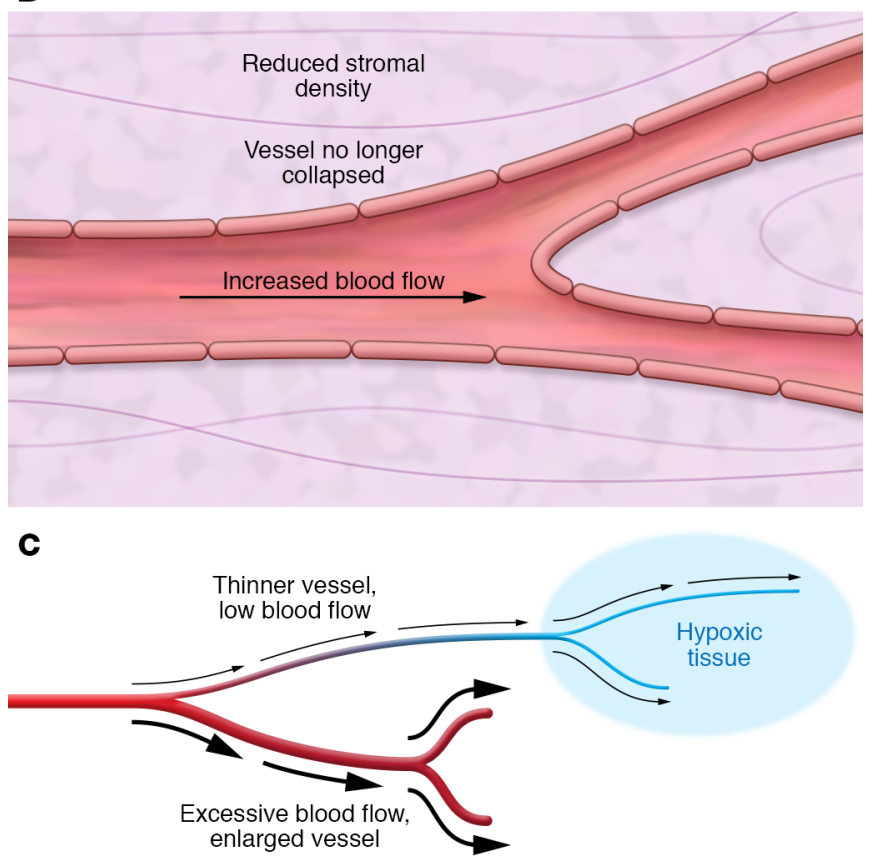

D

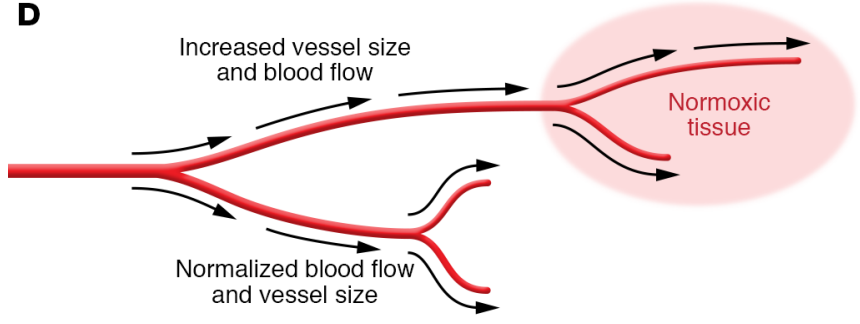

E

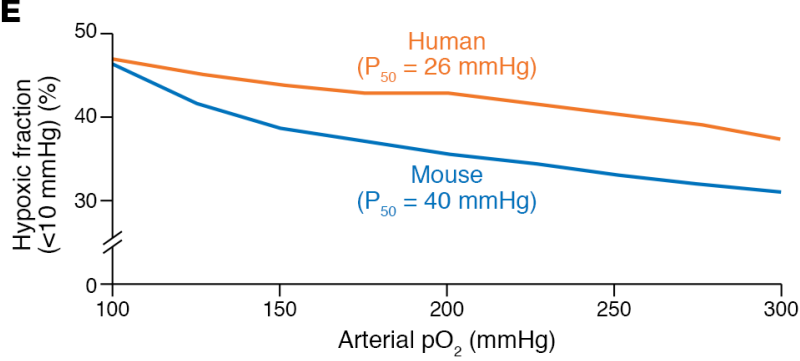

Figure 1. Methods to improve tumor perfusion and reduce hypoxia. (A and $\mathbf{B}$ ) Images showing how reduction in density of extracellular matrix can improve tumor perfusion and oxygen delivery. (C and D) Metronomic antiangiogenic therapy can normalize vasculature by promoting vascular maturation. Among other effects, this treatment can reduce disparities in vessel diameter at bifurcation points. Since flow resistance is proportional to the vessel radius raised to the fourth power, small differences in the diameter of daughter vessels leads to large effects on flow distribution. (C) Discrepancy in vessel diameters causes vascular hypoxia in a daughter vessel. (D) Normalization restores equal flow distribution to both daughter vessels, eliminating hypoxia. (E) Theoretical demonstration of relative efficiencies of tumor hypoxia reduction by varying breathing gas $\mathrm{O}_{2}$ concentration for human versus rodent hemoglobin. These simulations were done using a Green's function approach to calculate the oxygen field in a $3 \mathrm{D}$ region of a tumor growing in a skin-flap window chamber (17). Experimentally determined input variables include 3D vascular network structure, flow velocity and hematocrit of all vessel segments, vascular $\mathrm{pO}_{2}$ throughout the network, and oxygen consumption rate. Baseline arterial $\mathrm{pO}_{2}$ is $100 \mathrm{mmHg}$ under air breathing conditions. At baseline the hypoxic fraction (defined as $\mathrm{pO}_{2}<10 \mathrm{mmHg}$ ) is around $47 \%$ for both hemoglobins. As arterial $\mathrm{pO}_{2}$ increases, the hypoxic fraction decreases for both hemoglobins. However, the rate at which the hypoxic fraction drops is greater for rodent hemoglobin $\left(P_{50}=40 \mathrm{mmHg}\right)$, than human hemoglobin $\left(P_{50}=26 \mathrm{mmHg}\right)$. Note that complete elimination of hypoxia cannot be achieved at the clinical limit of 3 atmospheres $\mathrm{O}_{2}$ for either hemoglobin, but the relative difference in remaining hypoxia is higher for human hemoglobin. These results strongly suggest that solely increasing oxygen content of blood is insufficient to eliminate hypoxia. Combining high $\mathrm{O}_{2}$ content breathing with strategies such as those described in $\mathbf{A}-\mathbf{D}$ would be more likely to successfully mitigate hypoxia.

bined with treatments that increase oxygen availability. In a recent review, we considered a range of strategies that improve drug delivery (14). Many of these methods also have the potential to improve oxygen delivery. First, excessive extracellular matrix (ECM) production combined with high tumor and stromal cell density results in high tissue pressure in tumors that reduces drug delivery. Methods that reduce ECM pressure enable reperfusion of vasculature that is collapsed by tissue pressure, thus improving perfusion and oxygen delivery (14) (Figure 1, A and B). Several trials are underway using recombinant humanized multiply pegylated hyaluronidase (PEGPH2O) to degrade ECM to increase delivery of small and large molecule ther- 
apeutics (NCT03634332, NCT02563548, NCT03481920) A second method would be to "normalize" the tumor vascular network as a means to improve transport (Figure 1, C and D). Metronomic dosing of VEGF inhibitors leads to prolonged vascular normalization and resets the immune system to be more reactive toward tumors (15). Third, regular exercise improves tumor perfusion and reduces hypoxia in preclinical models (14).

Increasing $\mathrm{O}_{2}$ delivery by breathing hyperoxic gases or decreasing $\mathrm{O}_{2}$ consumption rate can also increase oxygen concentration in tumors. Hatfield et al. demonstrated that chronic breathing of $60 \% \mathrm{O}_{2}$ enhanced immune reactivity in mice by downregulating immunosuppressive extracellular adenosine, followed by several downstream immunomodulatory effects (16). While this approach appeared very promising preclinically, differences in the $\mathrm{P}_{50}$ of murine versus human hemoglobin lead us to predict that this approach will be less efficient in improving oxygen delivery to human tumors (Figure $1 \mathrm{E})$. An alternative strategy would be to reduce $\mathrm{O}_{2}$ consumption rate, as reducing $\mathrm{O}_{2}$ consumption rate reduces hypoxia several fold more efficiently than breathing hyperoxic gases (17). Metformin, a drug for treating type 2 diabetes, is an example that works quite efficiently to reduce $\mathrm{O}_{2}$ consumption rate in tumors (18).

In summary, we have provided a rationale for considering the assessment of hypoxia and its modification for precision medicine and immunotherapy trials. As we have discussed previously, conduct of such studies should include methods to assess the extent of hypoxia at baseline and during treatment, in order to quantify the extent of hypoxia amelioration that is achieved (19). The degree of hypoxia reduction will likely relate to the efficacy of the therapy delivered.

Address correspondence to: Mark Dewhirst, Radiation Oncology Department, Box 3455 Duke University Medical Center, Room 201 MSRB, Research Drive, Durham, North Carolina 27710, USA. Phone: 919.684.4180;

Email:mark.dewhirst@duke.edu.

1. Collins DC, Sundar R, Lim JSJ, Yap TA. Towards precision medicine in the clinic: from biomarker discovery to novel therapeutics. Trends Pharmacol Sci. 2017;38(1):25-40.

2. Wang RF, Wang HY. Immune targets and neoantigens for cancer immunotherapy and precision medicine. Cell Res. 2017;27(1):11-37.

3. Lu Y, Liu Y, Oeck S, Glazer PM. Hypoxia promotes resistance to EGFR inhibition in NSCLC cells via the histone demethylases, LSD1 and PLU-1. Mol Cancer Res. 2018;16(10):1458-1469.

4. Rebucci M, Michiels C. Molecular aspects of cancer cell resistance to chemotherapy. Biochem Pharmacol. 2013;85(9):1219-1226.

5. Dewhirst MW, Cao Y, Moeller B. Cycling hypoxia and free radicals regulate angiogenesis and radiotherapy response. Nat Rev Cancer. 2008;8(6):425-437.

6. Corzo CA, et al. HIF-1 $\alpha$ regulates function and differentiation of myeloid-derived suppressor cells in the tumor microenvironment. J Exp Med. 2010;207(11):2439-2453.

7. Noman MZ, Janji B, Berchem G, Chouaib S. miR-210 and hypoxic microvesicles: two critical components of hypoxia involved in the regulation of killer cells function. Cancer Lett. 2016;380(1):257-262.

8. Westendorf AM, et al. Hypoxia enhances immunosuppression by inhibiting CD4+ effector
T cell function and promoting Treg activity. Cell Physiol Biochem. 2017;41(4):1271-1284.

9. Sethumadhavan S, et al. Hypoxia and hypoxiainducible factor (HIF) downregulate antigenpresenting MHC class I molecules limiting tumor cell recognition by T cells. PLOS ONE. 2017;12(11):e0187314.

10. Noman MZ, et al. PD-L1 is a novel direct target of HIF-1 $\alpha$, and its blockade under hypoxia enhanced MDSC-mediated T cell activation. J Exp Med. 2014;211(5):781-790.

11. Gaber T, et al. CTLA-4 Mediates inhibitory function of mesenchymal stem/stromal cells. Int J Mol Sci. 2018;19(8):E2312.

12. Jayaprakash $\mathrm{P}$, et al. Targeted hypoxia reduction restores $\mathrm{T}$ cell infiltration and sensitizes prostate cancer to immunotherapy. J Clin Invest. 2018;128(11):5137-5149.

13. Overgaard J, Horsman MR. Modification of hypoxia-induced radioresistance in tumors by the use of oxygen and sensitizers. Semin Radiat Oncol.1996;6(1):10-21.

14. Dewhirst MW, Secomb TW. Transport of drugs from blood vessels to tumour tissue. Nat Rev Cancer. 2017;17(12):738-750.

15. Huang Y, et al. Vascular normalizing doses of antiangiogenic treatment reprogram the immunosuppressive tumor microenvironment and enhance immunotherapy. Proc Natl Acad Sci US A. 2012;109(43):17561-17566.

16. Hatfield SM, et al. Immunological mechanisms of the antitumor effects of supplemental oxygenation. Sci Transl Med. 2015;7(277):277ra30.

17. Secomb TW, Hsu R, Braun RD, Ross JR, Gross JF, Dewhirst MW. Theoretical simulation of oxygen transport to tumors by three-dimensional networks of microvessels. In: Hudetz AG, Bruley DF, eds. Oxygen Transport to Tissue XX. Advances in Experimental Medicine and Biology, vol 454. Boston, Massachusetts, USA: Springer; 1998:629-634.

18. Chowdhury S, et al. MATE2 Expression is associated with cancer cell response to metformin. PLOS ONE. 2016;11(12):e0165214.

19. Dewhirst MW. A potential solution for eliminating hypoxia as a cause for radioresistance. Proc Natl Acad Sci U S A. 2018;115(42):10548-10550. 